\title{
Effect evaluation on a medical device for drinking water filtration designed for reduction of microbial density in areas at risk
}

\author{
Lucia Bonadonna, Claudia Cataldo, Maurizio Semproni \\ Dipartimento di Ambiente e Connessa Prevenzione Primaria, Istituto Superiore di Sanità, Roma
}

Key words: Drinking water, Filtration systems, Legionella, Microorganisms, Removal, Faucet

Valutazione dell'effetto di un dispositivo per la filtrazione di acqua di rete per l'abbattimento delle densità microbiche in ambienti comunitari

\section{SUMMARY}

In areas at risk (hospitals, nursing homes) but in also in community such as schools, swimming pools, gyms, drinking water plumbing system can harbor also pathogenic microrganisms, potential vehicles of diseases because of structural factors and phenomena of microbial regrowth. In a setting of prevention, for limiting the exposure to pathogens in the environment and for minimizing the risk, at the point of use, specific disposable filter units could be installed.

Aims. The purpose of this study was to examine the effectiveness in retaining microrganisms of a medical devices installed at drinking water faucets.

Materials and methods. One hundred and eighty samples of water were microbiologically analyzed. Natural heterotrophic microbial flora present in drinking water were analytically determined before and after the passage through the system. Contemporaneously, twenty four suspensions of Pseudomonas aeruginosa and Escherichia coli at known concentration were determined for the calculation of the limit of retention calculated taking into examination the worst cases associated with the highest microbial concentrations.

Results. The investigation showed that the devices were both efficient at reducing the natural microbial load and at guaranteeing the removal of high concentration of microrganisms (till $5 \mathrm{log}$ ). Fifty-seven out of 90 samples analyzed for the heterotrophs did not show any growth after the drainage through the system. In the positive samples, $86 \%$ showed a microbial concentration ranging between I e $3 \mathrm{CFU} / \mathrm{L}$. Progressive growing concentrations of $P$. aeruginosa and $E$. coli proved a high limit of retention: none growth was obtained till the concentrations of $1.3 \times 10^{12} \mathrm{CFU} / \mathrm{mL}$ and $5.0 \times 10^{13} \mathrm{CFU} / \mathrm{mL}$, respectively.

Conclusions. The data show that the filter units installed directly at the point of use can be helpful at guaranteeing reliable retention of microbes. Still, in order to obtain an appropriate operation of the device, in spite of both the ease of use and safety margin, it is necessary to implement an efficient and orderly activity of maintenance according to the manufacturer's instructions.

\section{INTRODUZIONE}

Nelle acque utilizzate a scopo potabile la flora microbica costituisce una presenza costante. Infatti, se è pur vero che l'acqua sottoposta a trattamenti di potabilizzazione è microbiologicamente diversa e igienicamente più sicura rispetto a quella grezza, essa tuttavia può ancora contenere e trasportare microrganismi che fanno parte della flora ambientale naturale (eterotrofi, Legionella, micobatteri) - senza costituire una specifica contaminazione - e, occasionalmente, microrganismi derivati da contaminazioni esterne o sopravvissuti ai processi di trattamento delle acque (es., microrganismi che producono forme di resistenza) (1).

È noto che i trattamenti delle acque, compresa la disinfezione, hanno lo scopo di eliminare la componente microbica, che può anche includere microrganismi patogeni. Tuttavia, è anche vero che i processi di trattamento esercitano una pressione selettiva sui microrganismi presenti nell'acqua. Molti di essi sono in grado di sviluppare strategie per la sopravvivenza; ad esempio una vita allo stato quiescente della cellula batterica danneggiata e metabolicamente ancora attiva ma non più capace di moltiplicarsi in coltura.

Durante il percorso nel sistema di distribuzione e fino ai rubinetti, i microrganismi possono comunque venirsi a trovare in condizioni fisiologiche e spaziali favorevoli e, grazie alla capacità di adesione alle strutture e ai materiali della rete idrica, partecipare alla formazione di biofilm $(3,4)$.

In questo assetto, i microrganismi presenti in rete sono in grado di sopravvivere e, in condizioni favorevoli, anche di moltiplicarsi (9). Sito di con-

\section{Corresponding author: Lucia Bonadonna}

Dipartimento di Ambiente e Connessa Prevenzione Primaria, Istituto Superiore di Sanità

Viale Regina Elena, 299 - 0016 I Roma - Tel.: 0649902317 - Fax: 0649902390

E-mail: lucybond@iss.it 
centrazione di nutrienti e con effetto protettivo verso i disinfettanti utilizzati nella potabilizzazione, il biofilm è anche una nicchia potenziale per il trasferimento di caratteri di virulenza e resistenza agli antibiotici (12).

I biofilm microbici si formano sulle superfici a contatto con l'acqua di condotte, serbatoi e dispositivi di erogazione. La presenza di carbonio organico ne favorisce lo sviluppo che, per lo più, si rileva dove è ridotta la velocità di flusso dell'acqua (diramazioni, curve, raccordi, valvole) o dove esiste un potenziale ristagno (bracci morti, tubi delle utenze private, rubinetti, soffioni, guarnizioni, raccordi e rompigetto). In condizioni di dinamicità del sistema, i biofilm si distaccano dal substrato e, trasportati dall'acqua, giungono ai rubinetti dei consumatori.

I biofilm degli acquedotti possono ospitare un'ampia varietà di organismi patogeni e potenziali patogeni, un rischio reale per la salute dei consumatori se si considera che un distacco di cellule microbiche, e l'adesione di altre, sarebbe osservabile in continuo $(6,10)$.

Negli ambienti ospedalieri e nelle case di riposo per anziani, ove pazienti e ospiti sono sovente affetti da deficit immunitari, la rete idrica può quindi diventare una delle maggiori fonti di infezione per la sua capacità di essere una potenziale riserva di patogeni primari e opportunisti (2). Numerosi microrganismi - in particolare, Legionella, Pseudomonas aeruginosa, micobatteri, protozoi - presenti nei biofilm, diffusi attraverso l'acqua e aerosolizzati nell'ambiente, costituiscono un problema di grande impatto sanitario. In questo tipo di ambienti, spesso proprio la vetustà del sistema idrico, la presenza di tratti ciechi e le difficoltà di bonifica - attività economicamente impegnativa - rendono improbabile l'eradicazione permanente delle nicchie di proliferazione microbica (5). Inoltre è noto che, anche dopo il risanamento e il ripristino di condizioni idonee, $\mathrm{i}$ requisiti di qualità dell'acqua possono di nuovo peggiorare, anche a breve distanza di tempo. È per questo che, a scopo di prevenzione sanitaria, si può tentare di ridurre la diffusione di patogeni nell'ambiente e minimizzare il rischio di malattie idrotrasmesse mediante l'installazione diretta di specifici filtri ai rubinetti dei servizi igienici e ai soffioni delle docce in ambienti ad alto rischio.

È stata quindi valutata la capacità di un dispositivo filtrante di questo tipo nel trattenere specie microbiche naturalmente presenti nelle acque potabili o artificialmente inoculate sotto forma di sospensioni a concentrazioni note.

\section{MATERIALI E METODI \\ Materiali}

Per verificare le potenzialità di riduzione delle concentrazioni microbiche in acque potabili in uscita da rubinetti sono state utilizzate capsule filtranti Sartobran P (Sartorius Stedim Biotech, Italia) applicate ai rubinetti di un sistema idrico simulandone normali condizioni d'uso.

Le capsule impiegate sono dispositivi certificati secondo la Direttiva del Consiglio Europeo 93/42/EEC e costituite da una doppia membrana con porosità nominali rispettivamente di $0.45 \mathrm{e}$ $0.22 \mathrm{~mm}$, con una superficie di filtrazione effettiva di $500 \mathrm{~cm}^{2}$. In condizioni ordinarie e di uso corretto, la capsula può essere utilizzata per un massimo di tre giorni consecutivi, sottoposta successivamente a sterilizzazione e riposizionata al punto d'uso. La ditta produttrice garantisce che l'integrità e le prestazioni della capsula si mantengono fino a 40 cicli di sterilizzazione.

\section{Procedure analitiche}

Per valutare le prestazioni dei dispositivi filtranti utilizzati nella nostra indagine sono state effettuate analisi microbiologiche di acqua di rubinetto tal quale e in uscita della capsula applicando le procedure di seguito descritte. Ogni campione di acqua prelevato in uscita dalla capsula era associato al prelievo di un campione di acqua che, dallo stesso rubinetto, non attraversava la capsula. L'esame di entrambi i campioni era eseguito con le stesse procedure analitiche.

I microrganismi presi in considerazione durante l'indagine appartenevano al gruppo dei batteri eterotrofi e alle specie Pseudomonas aeruginosa ed Escherichia coli.

\section{Capsula in uso continuo}

Una capsula è stata installata ad un rubinetto servito da acqua potabile clorata della rete idrica pubblica. Seguendo le raccomandazioni della casa produttrice, la capsula è stata mantenuta in uso per 3 giorni consecutivi tra una sterilizzazione e la successiva, quindi autoclavata a $121^{\circ} \mathrm{C}$ per 15 minuti e riposizionata al punto d'uso. Nello studio svolto le fasi di sterilizzazione sono state protratte per 45 cicli per verificarne l'efficienza oltre il periodo consigliato dal produttore (40 cicli), per un totale di $90+90$ campioni di acqua analizzati. La procedura operativa appena descritta si è protratta per sei mesi ed è stata svolta secondo un protocollo che prevedeva diverse fasi tra loro correlate:

i) determinazione quantitativa degli eterotrofi a $22^{\circ} \mathrm{C}$ direttamente nell' acqua del rubinetto;

ii) successiva filtrazione di $70 \mathrm{~L}$ di acqua attraverso la capsula riposizionata allo stesso rubinetto;

iii) prelievo di $1 \mathrm{~L}$ di acqua per la determinazione dello stesso parametro.

Il giorno successivo, $70 \mathrm{~L}$ di acqua venivano fatti fluire attraverso la capsula senza procedere all'e- 
secuzione dell'analisi, simulando quindi un consumo medio giornaliero.

Trascorse 72 ore dall'analisi, corrispondenti al terzo giorno di uso della capsula, dopo aver filtrato altri $70 \mathrm{~L}$ attraverso la capsula, si è proceduto al prelievo di $1 \mathrm{~L}$ di acqua ed alla sua immediata analisi come in precedenza descritto. Con questa procedura continuata nel tempo, è stata pertanto verificata, sulla base dei valori di carica microbica ottenuti, l'efficienza di filtrazione della capsula e la sua capacità di trattenere i microrganismi con un uso ripetuto e ad intervalli di 3 giorni, come raccomandato dalla ditta produttrice.

\section{Determinazione del limite di ritenzione della capsula}

Contemporaneamente, in due distinte capsule filtranti installate a rubinetti, sono state iniettate attraverso la valvola di sfiato, situata a monte sulla capsula, aliquote separate e crescenti di sospensioni microbiche di Pseudomonas aeruginosa ed Escherichia coli (intervalli di concentrazione tra $10^{2} \mathrm{UFC} / \mathrm{mL}$ a $10^{14} \mathrm{UFC} / \mathrm{mL}$ ). Dopo inoculo di $1 \mathrm{~mL}$ di ciascuna coltura batterica a concentrazione nota attraverso la valvola di sfiato, 1 $\mathrm{L}$ d'acqua è stato fatto passare attraverso la capsula, prelevato e analizzato immediatamente con i metodi descritti di seguito. L'eventuale sviluppo di colonie sul terreno di crescita avrebbe segnalato la perdita della capacità di ritenzione del filtro.

\section{Metodi analitici}

Conteggio degli eterotrofi. Per l'analisi dell'acqua di rete tal quale e in uscita dalla capsula filtrante sono stati analizzati un totale di 180 campioni prelevati asetticamente e addizionati con tiosolfato di sodio. Delle acque tal quali sono stati analizzati $0.1 \mathrm{~mL}$ e $1 \mathrm{~mL}$ mentre, delle acque in uscita, prevedendone una migliore qualità, è stato analizzato 1 L. La tecnica analitica adottata è stata quella della filtrazione su membrane di acetato di cellulosa (porosità nominale di $0.45 \mu \mathrm{m}$ ) (Millipore). Le membrane sono state quindi poste sul terreno non selettivo Water Plate Count Agar (WPC) (Oxoid), incubato a $22^{\circ} \mathrm{C}$ per 7 giorni per la determinazione dei batteri eterotrofi.

Sospensioni batteriche a concentrazione nota. Per la verifica del limite di ritenzione del dispositivo rispetto a microrganismi a concentrazione nota e intenzionalmente elevata, sono stati selezionati Pseudomonas aeruginosa ATCC 9027 (intervallo di concentrazione: $10^{2}, 10^{12} \mathrm{UFC} / \mathrm{mL}$ ) ed Escherichia coli ATCC 8739 (intervallo di concentrazione: $10^{2}, 10^{14} \mathrm{UFC} / \mathrm{mL}$ ).

Colture pure dei due ceppi sono state risospese in $20 \mathrm{~mL}$ di Trypticase Soy Broth (TSB) (BBL) ed il brodo incubato a $37^{\circ} \mathrm{C}$ per 24 ore. Per determinare la concentrazione dei batteri vitali nelle diluizioni decimali sono state seminate aliquote note sul terreno colturale non selettivo Tryptic Soy Agar (TSA) (Difco) incubato a $37^{\circ} \mathrm{C}$ per 24 ore per il ceppo di $E$. coli e a $37^{\circ} \mathrm{C}$ per 48 ore per Pseudomonas aeruginosa. Per l'esame dei campioni all'uscita dalla capsula è stato raccolto $1 \mathrm{~L}$ del filtrato, suddiviso in due sub-campioni analizzati successivamente mediante la tecnica della filtrazione su membrana (acetato di cellulosa, porosità nominale di $0.45 \mu \mathrm{m}$ ). Ogni membrana è stata posta sul terreno colturale Tryptic Soy Agar (TSA) (Difco) ed incubata a $37^{\circ} \mathrm{C}$ per 24 ore per la ricerca di E. coli e per 48 ore per quella di Pseudomonas aeruginosa.

\section{RISULTATI}

L'analisi dei 90 campioni di acqua tal quale ha fornito conteggi di eterotrofi molto variabili, con valori compresi tra $13 \mathrm{UFC} / \mathrm{mL}$ e $2610 \mathrm{UFC} / \mathrm{mL}$ e concentrazioni medie di $122 \mathrm{UFC} / \mathrm{mL}$ (DS 313).

Per lo stesso parametro, l'esame dei 90 campioni dopo passaggio attraverso l'unità filtrante ha fornito risultati negativi per il $57 \%$ dei campioni e risultati positivi per il restante $43 \%$. Di questi, 1' $86 \%$ presentava conteggi tra 1 e $3 \mathrm{UFC} / \mathrm{L}$. Valori di concentrazione variabili tra 3 e $6 \mathrm{UFC} / \mathrm{L}$ sono stati osservati nel $7 \%$ dei campioni, mentre il $6 \%$ ha mostrato concentrazioni variabili tra $6 \div 300$ $\mathrm{UFC} / \mathrm{L}$. Valori più elevati $(>300 \mathrm{UFC} / \mathrm{L})$ sono stati riscontrati nell' $1.2 \%$ del totale.

Nella Figura I viene mostrato l'andamento dei risultati positivi/negativi relativi alla determinazione degli eterotrofi rilevati all'uscita dalla

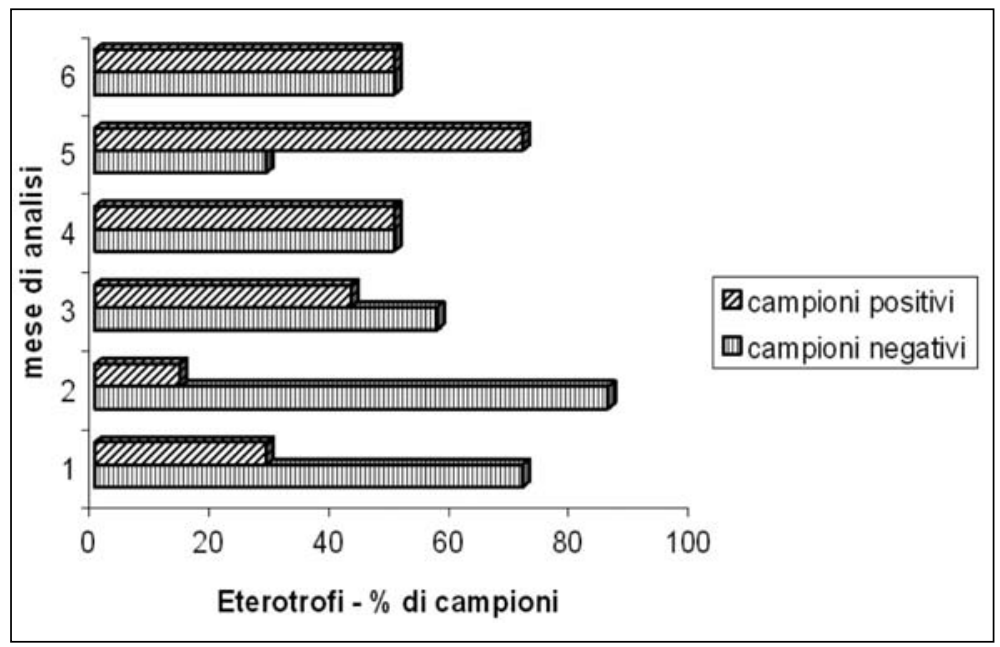

Figura I. Risultati relativi ai campioni positivi/negativi per gli eterotrofi: andamento delle prestazioni della capsula lungo l'intero periodo d'uso. 
capsula mantenuta in uso continuo.

Appare interessante osservare che, dopo il secondo mese di impiego, la percentuale di campioni negativi si riduce con conseguente diminuzione del numero di campioni positivi. D'altronde, durante il quarto e il sesto mese di studio, il numero di campioni positivi è risultato essere pari a quello dei campioni negativi. Sebbene sia implicito concludere che l'esiguità delle conte residue non comprometta il buon esito della filtrazione, sembra ragionevole assistere, nel tempo, ad una riduzione delle prestazioni del dispositivo filtrante che si manifesta con la perdita della capacità di trattenere i microrganismi presenti nell'acqua.

Tuttavia, iniettando a monte del filtro concentrazioni progressivamente crescenti di P. aeruginosa ed E. coli, il limite di ritenzione della capsula è molto elevato. In questo caso, risultati negativi sono stati ottenuti fino alle concentrazioni di $1.3 \times 10^{12} \mathrm{UFC} / \mathrm{mL}$ e di $5 \times 10^{13} \mathrm{UFC} / \mathrm{mL}$, rispettivamente. A concentrazioni superiori ( $P$. aeruginosa $2.6 \times 10^{12} \mathrm{UFC} / \mathrm{mL}$ ed $E$. coli $\left.1 \times 10^{14} \mathrm{UFC} / \mathrm{mL}\right)$, il filtro non appariva più in grado di trattenere $\mathrm{i}$ microrganismi che pertanto risultavano presenti nell' acqua filtrata e si sviluppavano quindi sul terreno di crescita.

\section{DISCUSSIONE}

L'installazione di dispositivi di filtrazione dell'acqua ai rubinetti e ai soffioni delle docce degli impianti idrici può trovare applicazione quando, in assenza di altre specifiche misure correttive o per loro scarsa efficacia, è necessario mettere in sicurezza punti d'uso in ambienti a rischio o dove sono segnalati persistenti problemi di qualità dell'acqua. Nello studio presentato è stata valutata la capacità della capsula Sartobran P di trattenere i microrganismi presenti sia naturalmente nell'acqua distribuita in rete sia artificialmente iniettati a concentrazioni elevate nel sistema filtrante, per simulare condizioni estreme di contaminazione e definire il limite di ritenzione della capsula.

Il corretto impiego della capsula, come prescritto dalla ditta produttrice, prevede un periodo d'uso di tre giorni consecutivi prima della sua disinstallazione, sterilizzazione e riutilizzo, per un periodo massimo di utilizzazione di quaranta cicli. Dalle prove effettuate, superando anche il numero di cicli consigliato, è stato osservato che il filtro ha mantenuto prestazioni elevate. Infatti, con riferimento ad $1 \mathrm{~L}$ di acqua filtrata, le percentuali di abbattimento delle concentrazioni microbiche rispetto all'acqua tal quale sono state calcolate mediamente pari al $99.99 \%$. Inoltre, se per il 57\% del totale dei campioni analizzati per gli eterotrofi non è stata riscontrata alcuna crescita, per i campioni risultati positivi, le analisi hanno mostrato valori di abbattimento di $3 \div 5$ unità logaritmiche. Anche considerando il volume totale di acqua fatto fluire attraverso il filtro $(210 \mathrm{~L})$ ad ogni ciclo, la capsula ha dimostrato una buona capacità nel trattenere $\mathrm{i}$ batteri anche dopo il superamento del periodo di impiego raccomandato dal produttore che sembra quindi offrire un buon margine di sicurezza per il buon funzionamento del dispositivo. È comunque evidente che, con il passare del tempo, la capacità filtrante diminuisca, anche se il numero di microrganismi in grado di attraversare il filtro rimane, in maniera relativamente costante, molto ridotto. Infatti, solo il 7.2\% dei campioni analizzati ha fornito valori superiori a $6 \mathrm{UFC} / \mathrm{L}$.

Anche al passaggio di sospensioni batteriche contenenti concentrazioni molto elevate, il sistema si è dimostrato in grado di trattenere microrganismi, con limiti di ritenzione estremamente elevati $\left(10^{12}\right.$ $\mathrm{UFC} / \mathrm{ml}$ per $P$. aeruginosa e di $10^{13} \mathrm{UFC} / \mathrm{ml}$ per $E$. coli).

Processi di trattamento e impiego di disinfettanti, generalmente alogenati, rappresentano fasi necessarie per rendere un'acqua idonea all'uso potabile. La disinfezione dell'acqua è la barriera finale, sia per la sua potabilizzazione, sia per il mantenimento delle sue caratteristiche igienico-sanitarie durante la sua distribuzione in rete. Tuttavia è difficile assicurare concentrazioni di disinfettanti adeguate fino ai punti d'uso degli impianti idrici e, soprattutto, è necessario considerare che, quando all'interno della rete è presente biofilm, colonizzazione, sopravvivenza e moltiplicazione di specie microbiche sono inevitabili.

Diversi e noti sono i fattori che concorrono alla colonizzazione microbica delle reti idriche. Tuttavia, risulta ancora difficile descrivere esattamente il ruolo della contaminazione microbica dell'acqua nella genesi delle infezioni, se non per Legionella e per alcuni micobatteri ambientali. In generale, è lecito affermare che la reale incidenza di infezioni è fortemente sottostimata per la persistente scarsa considerazione del problema, in ambito sia di comunità, sia ospedaliero. In letteratura, diversi casi di infezione e malattia per esposizione ad acqua contaminata da batteri ambientali quali Pseudomonas aeruginosa, Acinetobacter, Stenotrophomonas maltophilia, Mycobacterium, Chryseomonas e, soprattutto Legionella, associati a biofilm, sono stati segnalati in pazienti immunocompromessi $(7-8,13)$.

Gestire un impianto idrico potenzialmente colonizzato da microrganismi rappresenta un problema di difficile soluzione perché, anche quando sottoposto a trattamenti di bonifica, il mantenimento di idonee condizioni igieniche dell'acqua può non risultare duraturo (11). È noto, infatti, ad 
esempio, che la tolleranza ai disinfettati dei microrganismi costituenti i biofilm è di $2-3$ ordini di grandezza maggiore rispetto alle cellule microbiche libere nel flusso d'acqua (14).

In ambienti comunitari, come ospedali, case di cura, scuole, ecc. risulta quindi fondamentale svolgere attività di prevenzione che, oltre a controlli regolari della qualità dell'acqua, preveda lo svolgimento di interventi periodici programmati che assicurino il mantenimento di condizioni sanitarie idonee, sia limitando la possibilità di sviluppo di nicchie microbiche attraverso la pulizia degli impianti, sia rimuovendo i sedimenti dai serbatoi e dalle tubature e sostituendo giunti, filtri dei rubinetti e soffioni delle docce, tubi flessibili e ogni altro elemento di discontinuità.

Accanto alle ordinarie e straordinarie misure di controllo e gestione degli impianti idrici nelle strutture a rischio o dove non è possibile mantenere idonei requisiti di qualità dell'acqua, il posizionamento di capsule filtranti ai punti d'uso dell'impianto potrebbe costituire una misura di prevenzione integrativa, soprattutto in relazione alla diffusione di batteri quali Legionella e micobatteri che possono rappresentare, sebbene in misura diversa, un complesso problema comunitario di sanità pubblica (15).

L'indagine svolta ha dimostrato che i dispositivi sottoposti a verifica, oltre ad essere efficaci nell'eliminare la carica microbica naturale dell'acqua, hanno il vantaggio di garantire anche l'abbattimento di alte concentrazioni di microrganismi e quindi di essere sicuri, in casi di emergenza, per ogni tipologia di acqua erogata. A tutela e garanzia di un corretto funzionamento del sistema, nonostante la facilità d'uso, di applicazione e l'ampio margine di sicurezza, è comunque necessario mettere in opera un'efficace e sistematica attività di manutenzione sulla base delle condizioni stabilite dal costruttore.

\section{BIBLIOGRAFIA}

1. Allen M, Edberg S, Reasoner D. Heterotrophic plate count (HPC) bacteria - What is their significance in drinking water? In: Proceedings of the NFS International/WHO Symposium on Bacteria in drinking water. Public health implication? Geneve: WHO 2002.
2. Anaissie EJ, Penzak SR, Dignani C. The hospital water supply as a source of nosocomial infections: a plea for action. Arch Intern Med 2002; 162: 1483-92.

3. Block JC. Biofilms in potable water distribution systems. In: Melo LF, Bott TR, Fletcher M, Capdeville B, Eds. Biofilms: science and technology. London: Kluwer Academic Publishers, 1992.

4. Bonadonna L, Memoli G, Chiaretti G. Formazione di biofilm su materiali a contatto con acqua: aspetti sanitari e tecnologici. Roma: Istituto Superiore di Sanità; 2008; Rapporti ISTISAN 08/19.

5. Coccagna L. Trattamenti di bonifica e prevenzione. In Atti Convegno "Legionella spp: microbiologia ambientale e tutela della collettività". Bologna 14/12/1999

6. Costerton JW, Stewart PS, Greenberg EP. Bacterial biofilms: a common cause of persistent infections. Science 1999; 284: 1318-22.

7. Dykewicz CA. Hospital infection control in hematopoietic stem cell transplant recipients. Emerg Infect Dis 2001; 7: 263-67.

8. Glasmacher A, Engelhart S, Exner M. Infections from HPC organisms in drinking water amongst the immunocompromised. In: Bartram J, Ed. Heterotrophic plate counts and drinking-water safety. London: IWA Publishing, 2003.

9. Lehtola MJ, Miettinen IT, Keinanen MM, et al. Microbiology, chemistry and biofilm development in a pilot drinking water distribution system with copper and plastic pipes. Water Res 2004; 38: 3769-79.

10. Morris RD, Levin R. Estimating the incidence of waterborne infectious disease related to drinking water in the United States. In: Reichard EG, Zapponi GA, Eds. Assessing and managing health risk from drinking water contamination: approaches and applications. Wallingford: IAHS, 1995.

11. Muraca P, Stout JE, Yu VL. Comparative assessment of chlorine, heat, ozone, and UV light for killing Legionella pneumophila within a model plumbing system. Appl Environ Microbiol 1987; 53: 447-53.

12. Muscarella LF. Contribution of tap water and environmental surfaces to nosocomial transmission of antibiotic-resistant Pseudomonas aeruginosa. Infect Cont Hosp Epidemiol 2004; 25: 342-5.

13. Oren I, Zuckerman T, Aviv I, Finkelstein R, Yigla M, Rowe JM. Nosocomial outbreak of Legionella pneumophila serogroup 3 pneumonia in a new bone marrow transplant unit: evaluation, treatment and control. Bone Marrow Transplant 2002; 30: 175-9.

14. Stout JE, Yu VL. Experiences of the first 16 hospitals using copper-silver ionization for Legionella control: implications for the evaluation of other disinfection modalities. Infect Control Hosp Epidemiol 2003; 24: 563-8.

15. World Health Organization. Legionella and the prevention of legionellosis. Geneve: WHO, 2007. 